\title{
Chest computed tomography signs associated with pejorative evolution in COVID-19 patients
}

\author{
Romain Auger ${ }^{1, A, B, C, D, E, F}$, Paul-Armand Dujardin 2,A,C,D,E , Aurore Bleuzen ${ }^{1, A}$, Juliette Buraschi ${ }^{1, A}$, Natacha Mandine ${ }^{1, B}$, \\ Sylvain Marchand-Adam ${ }^{3, D}$, Arthur Pearson ${ }^{1, A, C, D, E}$, Gaëlle Derot ${ }^{1, A, B, D, E, F}$ \\ 'Department of Radiology, Centre Hospitalier Régional Universitaire de Tours, France \\ ${ }^{2} \mathrm{CIC}$ 1415, Centre Hospitalier Régional Universitaire de Tours, Inserm, Tours Cedex, France \\ ${ }^{3}$ Department of Respiratory Medecine, Centre Hospitalier Régional Universitaire de Tours, France
}

\section{Abstract}

Purpose: The aim of this study was to evaluate how chest computed tomography (CT) can predict pejorative evolution in COVID-19 patients.

\begin{abstract}
Material and methods: Data on 349 consecutive patients who underwent a chest CT either for severe suspected COVID-19 pneumonia or clinical aggravation and with COVID-19 were retrospectively analysed. In total, 109 had laboratory-confirmed COVID-19 infection by a positive reverse-transcription polymerase chain reaction (RT-PCR) and were included. The main outcomes for pejorative evolution were death and the need for invasive endotracheal ventilation (IEV). All the CT images were retrospectively reviewed, to analyse the CT signs and semiologic patterns of pulmonary involvement.
\end{abstract}

Results: Among the 109 COVID-19 patients, 73 (67\%) had severe symptoms of COVID-19, 28 (25.7\%) needed an IEV, and $11(10.1 \%)$ died. The following signs were significantly associated with both mortality and need for IEV: traction bronchiectasis and total affected lung volume $\geq 50 \%(\mathrm{p}<10-3)$. Other CT signs were only associated with the need of IEV: vascular dilatation, air bubble sign, peribronchovascular thickening, interlobular thickening, and number of involved lobes $\geq 4$ ( $\mathrm{p}<10-3)$.

Conclusions: On a chest CT performed during the first week of the symptoms, the presence of traction bronchiectasis and high values of affected lung volume are associated with the need for IEV, and with mortality, in COVID-19 patients.

Key words: COVID-19, chest CT, IEV, critical care, bronchiectasis.

\section{Introduction}

Coronavirus disease (COVID-19) has been spreading worldwide since December 2019 from Wuhan, Hubei Province [1]. The virus called SARS-Cov-2 belongs to the Betacoronavirus genus, is composed of a positive singlestranded RNA, and has a 50-200 nm diameter [2,3]. Bats are the natural reservoir for coronaviruses [4]. By April $26^{\text {th }} 2020$, more than $2,355,853$ cases (of which 947,693 in
Europe) and 164,656 deaths worldwide had been reported due to this pandemic [5]. By March $16^{\text {th }} 2020$ in France, 124,114 cases had been confirmed, with 22,614 deaths [6]. Fever, cough, fatigue, diarrhoea, dyspnoea, and myalgia are the most common symptoms, but the patients' condition can deteriorate rapidly, requiring intensive medical care $[7,8]$. Real-time reverse-transcription polymerase chain reaction (RT-PCR) is the standard reference to detect viral nucleic acid, but false-negative results have

Correspondence address:

Romain Auger, Department of Radiology, Le Centre Hospitalier Régional Universitaire de Tours, Tours, France, e-mail: romain.auger100@gmail.com Authors' contribution:

A Study design · B Data collection · C Statistical analysis · D Data interpretation · E Manuscript preparation · F Literature search · G Funds collection 
been reported and have led to a $66-80 \%$ sensitivity due to multiple factors, notably the quality of sampling [9]. Compared to RT-PCR, chest computed tomography (CT) is an easy-to-use and a faster method to diagnose and assess an early pulmonary COVID-19 infection [10]. Chest CT can also help to monitor the evolution and diagnose complications of COVID-19. Indeed, some authors have demonstrated the diagnostic value of chest CT in COVID-19 pneumonia describing compatible radiological signs [11-13]. Ai et al. evaluated chest CT diagnostic value in 1014 cases of suspected COVID-19 using RT-PCR as a reference, and they determined a sensitivity of $97 \%$ and negative predictive value of $83 \%$ [10]. Few authors have studied the prognostic value of chest CT. Yuan et al. found in a small sample of 27 patients (of whom 10 died) higher rates of consolidation and air bronchogram in the dead patients' group [14]. In a retrospective study, Zhao et al. found pleural effusions and architectural distortions to be CT signs potentially correlated with severe deterioration in a small population of severe or deceased patients (14/101 patients) [15]. Yang et al. proposed a pulmonary inflammation load score, which was higher in patients with severe COVID-19 in comparison with patients who had a mild form of the disease [16]. However, despite these elements, few studies have shown whether there was a relation between some early chest CT signs and the need for invasive endotracheal ventilation (IEV).

In this study, our main objective was to assess whether some chest CT signs were associated with pejorative evolution (defined as a need for IEV or death), in COVID-19 patients.

\section{Material and methods}

\section{Study design}

Ethical approval of this monocentric retrospective study was delivered by the review board of our hospital. We used the data of a cohort of 349 patients included from March $19 \mathrm{t}^{\mathrm{h}}$ to April $28^{\text {th }} 2020$, which was created to study the diagnostic value of chest CT for COVID-19.

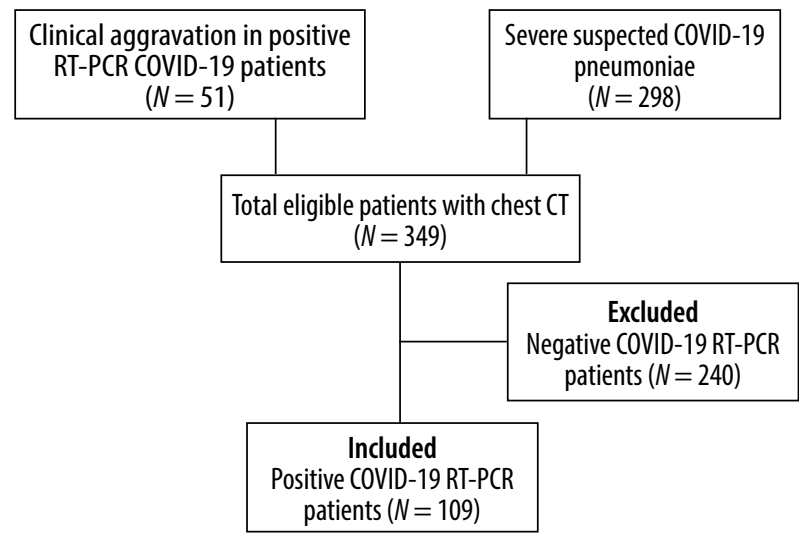

Figure 1. Flow-chart of the 109 patients included in the study
The patients were included in the cohort if they had a chest CT, either for suspected COVID-19 pneumoniae with severe symptoms or for clinical deterioration during a COVID-19 infection that had been confirmed with a positive RT-PCR. We included in our study 109 patients who had a positive COVID-19 RT-PCR.

The patient selection and inclusion process are represented in a flow diagram in Figure 1. The clinical severity scale was assessed according to the Chinese Centre of Disease Control and Prevention describing four groups of severity in the COVID-19 disease: uncomplicated illness, moderate pneumonia with dyspnoea, severe pneumonia, and acute respiratory distress syndrome (ARDS) $[17,18]$.

\section{RT-PCR test and clinical synthesis}

Coronavirus nucleic acid was collected with nasopharyngeal swab or/and bronchial aspiration. RT-PCR was performed using gene amplification RdRpE, and/or $\mathrm{N}$ (CNR Pasteur technic, AllPlex Seegene, Bosphore Anatolia; depending on availabilities). For patients who presented a high clinical probability of infection, two or three RT-PCR tests were performed if the previous results were negative, with a minimum of 48-72 hours between samples.

If one RT-PCR was positive, COVID-19 diagnosis was confirmed. In the case of multiple chest CTs, we used the one that was closest, timewise, to the first positive RT-PCR. All patients were included, whatever the time between positive RT-PCR and chest CT.

\section{Primary outcome}

The primary outcome was pejorative evolution, which we defined as use of IEV or death.

\section{Chest computed tomography protocol}

Chest CT was performed for all patients. Images were obtained in supine position by one of the two following CT scanners: an Aquilion Prime 160 (Toshiba, Japan) or a Somatom Force CT VB10 (Siemens Healthiners, Germany). The scanning parameters were as follows: tube voltage $120 \mathrm{kV}$, automatic dose modulation (30-210 mAs), matrix $512 \times 512$, pitch $0.35-0.99$, slice thickness $0.5 \mathrm{~mm}$, and field of view $500 \mathrm{~mm} \times 500 \mathrm{~mm}$. A $0.5 \mathrm{~mm}$ slice thickness was used after image reconstruction. When a pulmonary embolism was suspected, iodinated contrast media (300 mg/l) was intravenously injected (3 to $4 \mathrm{ml} / \mathrm{s}$ ) according to patient weight, with bolus tracking in the main pulmonary artery. Reconstructed images were archived and transferred to the informatic network.

\section{Chest computed tomography image analysis}

Images were reviewed by two radiologists, according to the consensual signs described in COVID-19 [7], and 
a final decision reached by consensus. Clinical history was available for interpretation.

Chest CT signs were analysed according to the Fleishner Society glossary [19]: ground glass-opacities (patchy, nodular or mixed), crazy paving (thickened intralobular and interlobular lines in a ground glass opacity), subpleural curvilinear bands, consolidation areas, air bubble sign, vascular dilatation, traction bronchiectasis, reticular interlobular thickening, pleural effusion, pleural thickening, number of involved lobes, inferior predominance, laterality, lung localisation (subpleural, central or dual distribution), compatible CT aspect of acute respiratory distress syndrom, compatible CT aspect of organizing pneumonia, compatible CT aspect of cardiac failure, lymphadenopathy, emphysematous lesions, bronchial thickening, endobronchial secretion, centrilobular nodule, and total affected lung volume $(0 \%,<10 \%, \geq 10-<25 \%, \geq 25-$ $<50 \%, \geq 50-<75 \%, \geq 75 \%)$. The cut-off values for lung volume involvement were chosen in accordance with the recommendations of the French Society of Radiology [20].

Compatible CT aspect of organising pneumonia (OP) was defined as consolidation (nodular, linear, perilobular, or peribronchovascular), subpleural curvilinear bands, halo sign, and/or atoll sign [21]. Traction bronchiectasis was defined as bronchial dilatations, with irregular contours, cylindrical, tubular, or fusiform, focalised in the areas affected by the ground glass opacities (GGO), consolidation, or crazy paving [22].

\section{Statistical analysis}

Statistical analysis was performed with $\mathrm{Matlab}^{\circledR} \mathrm{R} 2007 \mathrm{~b}$ (MathWorks Inc., Natick, USA). Qualitative variables were reported with number and percentage, and quantitative variables with mean and standard deviation.

In the included patients, we compared the frequency and extent of COVID-19 chest CT signs, between the deceased and survivor groups and between the IEV and non-IEV groups.

The followings chest CT signs and patterns were analysed: GGO, crazy paving, consolidation area, subpleural curvilinear bands, traction bronchiectasis, vascular dilatation, air bubble sign, interlobular thickening, total volume (50-100\% vs. < 50\%), compatible aspect of OP, compatible CT aspect of ARDS, distribution (subpleural, central, and dual - i.e. central and subpleural), inferior localisation, and number of involved lobes ( 0 to 3 vs. 4 or 5 involved lobes). For statistical analysis, the $50 \%$ total volume involvement cut-off and the number of involved lobes $(<4$ vs. $\geq 4)$ were chosen based on a previous study by Yang et al., which found that these cut-offs were associated with the severity of the disease [16].

Association between chest CT signs and pejorative evolution in COVID-19 patients was evaluated using vs. tests when applicable (all theorical numbers superior to 5) and Fisher's exact test otherwise. All test results with a $p$-value $<0.05$ were considered statistically significant.

\section{Results}

\section{Clinical and population characteristics}

From March $19^{\text {th }}$ to April $28^{\text {th }}, 2020$ a total of 109 patients with a positive RT-PCR were included in the study. Their clinical and demographic characteristics are shown in Table 1. Among them, 73 (67\%) had severe symptoms (severe pneumonia or ARDS as described above), 28 (25.7\%) had IEV, and $11(10.1 \%)$ died. The mean time interval between the RT-PCR test and chest CT was inferior to 1.5 days.

Table 1. Clinical and demographic characteristics of the 109 patients included

\begin{tabular}{|l|c|}
\hline Variable & Value \\
\hline Age (years) & $64.6 \pm 17.2$ \\
\hline Male sex & $61(55.6 \%)$ \\
\hline Comorbidity & $81(74.0 \%)$ \\
\hline \begin{tabular}{l} 
Clinical severity \\
\hline \multicolumn{1}{|l|}{ Mild }
\end{tabular} & $12(11.0 \%)$ \\
\hline \multicolumn{1}{|c|}{ Moderate } & $24(22.0 \%)$ \\
\hline \multicolumn{1}{|c|}{ Severe } & $46(42.2 \%)$ \\
\hline \multicolumn{1}{|c|}{ Mild ARDS } & $1(0.9 \%)$ \\
\hline Severe ARDS & $15(13.7 \%)$ \\
\hline ICUH & $11(10.1 \%)$ \\
\hline IEV & $39(35.7 \%)$ \\
\hline Death & $28(25.7 \%)$ \\
\hline Life status unknown at the end of the study & $11(10.1 \%)$ \\
\hline Number of unknown RT-PCR before CT & $11(10.1 \%)$ \\
\hline Average time between first symptom and CT (days) & $55(50.5 \%)$ \\
\hline $\begin{array}{l}\text { Average time between first symptom and CT } \\
\text { among patients in ICUH (days) }\end{array}$ & $6.2 \pm 5.1$ \\
\hline Average time between CT and RT-PCR (days) & $5.9 \pm 4.3$ \\
\hline $\begin{array}{l}\text { Average time between RT-PCR and CT if RT-PCR } \\
\text { results are not known (days) }\end{array}$ & $1.49 \pm 2.48$ \\
\hline $\begin{array}{l}\text { Average time between RT-PCR and CT if RT-PCR } \\
\text { results are known (days) }\end{array}$ & $2.11 \pm 2.38$ \\
\hline Average time between admission and CT (days) & $1.10 \pm 2.15$ \\
\hline
\end{tabular}

Qualitative values are indicated as number and percentages. Quantitative values are indicated as mean and standard deviation. Comorbidity corresponds to the following: age $>65$ years, chronic respiratory disease, dialysis, cardiac insufficiency NYHA 3 or 4, history of cardiac diseases (arterial hypertension, coronaropathy, stroke, cardiac surgery), cirrhosis ( $\geq$ Child B), diabetes with complications or requiring insulin therapy, immunosupression (chemotherapy, biotherapy, immunosuppressive corticotherapy, uncontrolled HIV or CD4 $<200 / \mathrm{mm}^{3}$, metastatic cancer, all types of graft), BMI $>40$, or pregnancy.

ARDS - acute respiratory distress syndrome, ICUH - intensive care unit hospitalisation, IEV - invasive endotracheal ventilation, RT-PCR - reverse-transcription polymerase chain reaction, CT - computed tomography. 
Table 2. Chest CT signs for the 109 positive COVID-19 patients

\begin{tabular}{|c|c|}
\hline Signs & Values \\
\hline GG0 & $98(89.9 \%)$ \\
\hline Crazy paving & $65(59.6 \%)$ \\
\hline Not systematised consolidation area & $39(35.8 \%)$ \\
\hline Subpleural curvilinear bands & $63(57.8 \%)$ \\
\hline Traction bronchiectasis & $45(41.3 \%)$ \\
\hline Vascular dilatation & $25(22.9 \%)$ \\
\hline Air bubble sign & $12(11.0 \%)$ \\
\hline Interlobular thickening & $14(12.8 \%)$ \\
\hline Compatible aspect of ARDS & $7(6.4 \%)$ \\
\hline Compatible aspect of OP & $59(54.1 \%)$ \\
\hline $\begin{array}{l}\text { Compatible aspect of cardiac additional } \\
\text { decompensation }\end{array}$ & $6(5.5 \%)$ \\
\hline Lymphadenopathy & $13(11.9 \%)$ \\
\hline Bronchial thickening & $13(11.9 \%)$ \\
\hline Endobronchial secretion & $7(6.4 \%)$ \\
\hline Centrilobular nodule & $7(6.4 \%)$ \\
\hline Pneumothorax & $2(1.8 \%)$ \\
\hline Pulmonary embolism & $6(5.5 \%)$ \\
\hline Emphysematous lesions & $5(4.6 \%)$ \\
\hline Systematised consolidation & $6(5.5 \%)$ \\
\hline Pleural effusion & $17(15.6 \%)$ \\
\hline Pleural thickening & $4(3.7 \%)$ \\
\hline Bilateral involvement & $102(93.0 \%)$ \\
\hline Unilateral involvement & $3(2.7 \%)$ \\
\hline Subpleural localisation & $104(95.4 \%)$ \\
\hline Central localisation & $48(44.0 \%)$ \\
\hline Dual distribution & $52(47.7 \%)$ \\
\hline Inferior localisation & $75(68.8 \%)$ \\
\hline Number of involved lobes & $4.2 \pm 1.4$ \\
\hline Volume (total affected lung) & $\begin{array}{c}0 \%: 5(4.6 \%) \\
<10 \%: 13(11.9 \%) \\
\geq 10-25 \%: 24(22.0 \%) \\
\geq 25-<50 \%: 31(28.4 \%) \\
\geq 50-75 \%: 16(14.7 \%) \\
\geq 75 \%: 20(18.4 \%)\end{array}$ \\
\hline No CT signs & $4(3.7 \%)$ \\
\hline
\end{tabular}

All signs are described with number and percentage, except the number of involved lobes which is described with mean and standard deviation.

CT - computed tomography, GGO - ground glass opacities, ARDS - acute respiratory distress syndrome, $\mathrm{OP}$ - organising pneumonia.

Patients generally had chest CT on the same day or on the day after hospitalisation and a mean of 6.2 days after the first symptoms. Table 2 presents the chest CT signs of the 109 COVID-19 patients who had a positive RT-PCR. Seven (6.4\%) patients did not show COVID-19 CT signs (normal CT or incompatible CT signs).
Table 3. Association between chest CT signs and use of IEV in comparison with the non-IEV group, among positive COVID-19 RT-PCR patients

\begin{tabular}{|l|c|c|c|}
\hline Signs & $\begin{array}{c}\text { IEV group } \\
(n=28)\end{array}$ & $\begin{array}{c}\text { Non-IEV } \\
\text { group } \\
(n=81)\end{array}$ & $p$-value \\
\hline GG0 & $26(92.2 \%)$ & $72(88.9 \%)$ & - \\
\hline Crazy paving & $19(67.9 \%)$ & $46(56.8 \%)$ & - \\
\hline $\begin{array}{l}\text { Not systematised } \\
\text { consolidation area }\end{array}$ & $13(46.4 \%)$ & $26(32.1 \%)$ & - \\
\hline Subpleural curvilinear bands & $12(42.6 \%)$ & $51(63 \%)$ & - \\
\hline Traction bronchiectasis & $23(82.1 \%)$ & $22(27.2 \%)$ & $<10^{-3}$ \\
\hline Vascular dilatation & $13(46.4 \%)$ & $12(14.8 \%)$ & $<10^{-3}$ \\
\hline Air bubble sign & $6(21.4 \%)$ & $6(7.4 \%)$ & 0.039 \\
\hline Interlobular thickening & $7(25 \%)$ & $7(8.6 \%)$ & 0.026 \\
\hline Compatible aspect of ARDS & $7(25 \%)$ & $0(0 \%)$ & $<10^{-3}$ \\
\hline Subpleural localisation & $28(100 \%)$ & $76(93.8 \%)$ & - \\
\hline Central localisation & $17(60.7 \%)$ & $31(38.3 \%)$ & - \\
\hline Dual distribution & $19(67.9 \%)$ & $33(40.7 \%)$ & - \\
\hline Inferior localisation & $20(71.4 \%)$ & $55(67.9 \%)$ & - \\
\hline Compatible aspect of OP & $13(46.4 \%)$ & $46(56.8 \%)$ & - \\
\hline $\begin{array}{l}\text { Peribronchovascular } \\
\text { thickening }\end{array}$ & $8(28.6 \%)$ & $6(7.4 \%)$ & $6.10^{-3}$ \\
\hline Number of involved lobes & $4.8(0.57)$ & $2.53(1.77)$ & $4.25 .10^{-3}$ \\
\hline Total affected volume $\geq 50 \%$ & $22(78.5 \%)$ & $15(18.5 \%)$ & $<10^{-3}$ \\
\hline
\end{tabular}

$\mathrm{CT}$ - computed tomography, $\mathrm{GGO}$ - ground glass opacities, IEV - invasive endotracheal ventilation, ARDS - acute respiratory distress syndrome, OP - organising pneumonia, RT-PCR reverse-transcription polymerase chain reaction, $\mathrm{ICUH}$ - intensive care unit hospitalisation. All signs are described with number and percentage, except the number of involved lobes which is described with mean and standard deviation. Dashes correspond to $p>0.05$.

\section{Computed tomography signs and pejorative evolution}

The frequency of chest CT signs and the calculated $p$-values of the corresponding $\chi^{2}$ tests can be found in Table 3 (comparing the need for IEV and no-IEV groups) and Table 4 (comparing the deceased and survivor groups).

Traction bronchiectasis and total affected lung volume $\geq 50 \%$ were significatively associated with mortality $\left(p=0.018\right.$, and $p<10^{-3}$, respectively), and IEV $\left(p<10^{-3}\right)$.

Vascular dilatation $\left(p<10^{-3}\right)$, air bubble sign ( $p=0.039)$, interlobular $(p=0.026)$ and peribronchovascular thickening $\left(p=6.10^{-3}\right)$, and the number of involved lobes $\geq 4\left(p<10^{-3}\right)$ were associated with the need for IEV.

\section{Discussion}

In our study, we found that traction bronchiectasis and total affected lung volume $\geq 50 \%$ (Figures $2 \mathrm{~A}, \mathrm{~B}$ and 3 ), were significantly associated with death, among positive RT-PCR COVID-19 patients. Also, vascular dilatation, traction bronchiectasis, and lesional expansion (total affected lung volume $\geq 50 \%$ or $\geq 4$ affected lobes) were 
Table 4. Association between chest computed tomography signs and death in comparison with the surviving group, among positive COVID-19 RT-PCR patients

\begin{tabular}{|l|c|c|c|}
\hline Signs & Deceased group $(n=11)$ & Surviving group $(n=87)$ & $p$-value \\
\hline GG0 & $11(100 \%)$ & $77(88.5 \%)$ & - \\
\hline Crazy paving & $7(63.6 \%)$ & $52(59.8 \%)$ & - \\
\hline Not systematised consolidation area & $4(36.4 \%)$ & $29(33.3 \%)$ & - \\
\hline Subpleural curvilinear bands & $4(36.4 \%)$ & $56(64.4 \%)$ & 0.018 \\
\hline Traction bronchiectasis & $8(72.7 \%)$ & $31(35.6 \%)$ & - \\
\hline Vascular dilatation & $3(27.3 \%)$ & $19(21.8 \%)$ & - \\
\hline Air bubble sign & $2(18.2 \%)$ & $8(9.2 \%)$ & - \\
\hline Interlobular thickening & $1(9.1 \%)$ & $12(13.8 \%)$ & - \\
\hline Compatible aspect of ARDS & $1(9.1 \%)$ & $3(3.4 \%)$ & - \\
\hline Subpleural localisation & $11(100 \%)$ & $82(94.3 \%)$ & - \\
\hline Central localisation & $6(54.5 \%)$ & $33(37.9 \%)$ & - \\
\hline Dual distribution & $6(54.5 \%)$ & $36(41.4 \%)$ & - \\
\hline Inferior localisation & $6(54.5 \%)$ & $61(70.1 \%)$ & - \\
\hline Compatible aspect of organizing & $4(36.4 \%)$ & $52(59.8 \%)$ & - \\
\hline pneumonia & $1(9.1 \%)$ & $9(10.3 \%)$ & - \\
\hline Peribronchovascular thickening & $4.4(0.36)$ & $2.54(1.76)$ & $<10^{-3}$ \\
\hline Number of involved lobes & $7(63.6 \%)$ & $23(26.4 \%)$ & - \\
\hline Total affected volume $\geq 50 \%$ & & & - \\
\hline
\end{tabular}

CT - computed tomography, GGO - ground glass opacities, RT-PCR - reverse-transcription polymerase chain reaction, ARDS - acute respiratory distress syndrome.

All signs are described with number and percentage, except the number of involved lobes, which is described with mean and standard deviation. Dashes correspond to $p>0.05$.
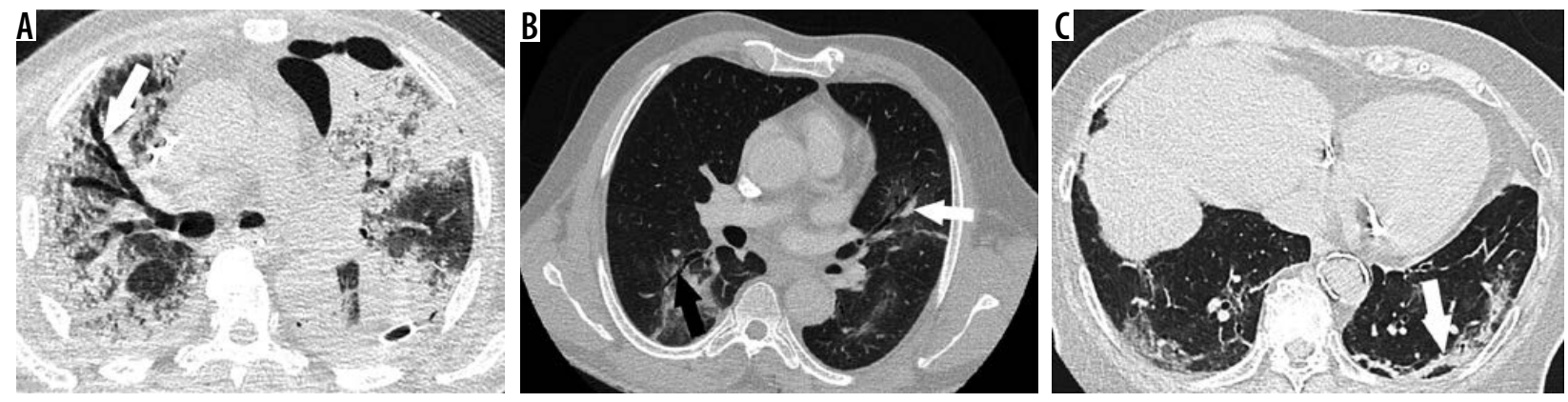

Figure 2. Chest computed tomography (CT) in positive reverse-transcription polymerase chain reaction COVID-19 cases. A) A 74-year-old man in intensive care unit hospitalisation (ICUH) with endotracheal ventilation, 11 days after first symptoms, massive traction bronchiectasis (white arrow) in the entire right lung, large areas of ground glass opacities (GGO) and subpleural consolidation, with $\geq 75 \%$ of total affected lung. Note a left anterior loculated pneumothorax and a left posterior pleural effusion drain. B) A 57-year-old man in ICUH, 13 days after first symptoms, dual distribution areas (subpleural and central) of GG0 in the lingula and the right inferior lobe with traction bronchiectasis (black arrow) and vascular dilatation (white arrow), with 50-75\% of total affected lung. C) A 67-year-old man in ICUH, 10 days after first symptoms, (T aspect of organised pneumonia (white arrow) with bilateral posterior subpleural curvilinear bands

significantly associated with IEV. Semiological CT anomalies like traction bronchiectasis, vascular dilatation, and bubble sign are consistent with indirect signs of acute pulmonary fibrosis, and they could explain the rapid clinical deterioration of some patients. Indeed, vascular dilatation could be due to endothelial lesions with inflammatory vasodilatation or maybe to parenchymal retractions associated with fibrosis. The air bubble sign is more frequent in the IEV group and could correspond to a bronchioloalveolar dilatation due to fibrosing damage or a previous existing cyst revealed by the diffuse infection [22]. Our findings are consistent with those of a study that had a similar sample size, i.e. in 60 patients showed that at least four affected lobes was correlated with death in critically ill patients [23]. Feng et al. also demonstrated that more lung lobes were involved in the severe and critical groups than in the moderate group; their findings are also consistent with our study [24].

We also observed patterns compatible with OP in more than $50 \%$ of patients (Figure $2 \mathrm{C}$ ). This concurs with a study by Copin et al. [25], which found an association between OP and fibrosis lesions in a pulmonary autopsy of a COVID-19 patient and described it as a specific histologic pattern of acute fibrinous and OP. 


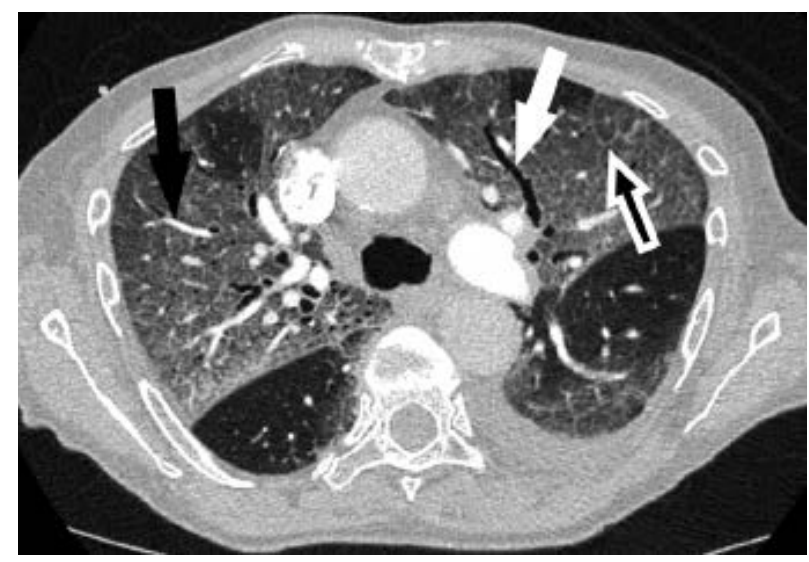

Figure 3. Chest computed tomography of a 76-year-old man in intensive care unit hospitalisation, 10 days after first symptoms, bilateral, large, and patchy ground glass opacities with crazy paving (white underlined dark arrow), traction bronchiectasis (white arrow) and vascular dilatation (dark arrow), with $50-75 \%$ of total affected lung

The significantly higher frequency of interlobular and peribronchovascular thickening in the IEV group could be due to alveolar interstitial oedema, similar to what can be observed in congestive heart failure, or due to the interstitial spreading of the inflammation [26]. However, unlike Zhao et al., lymphadenopathy and pleural effusion were not associated with a pejorative evolution in our cohort [15].

The frequency and type of chest CT signs are similar to previous studies except for consolidation and traction bronchiectasis, more prevalent in our study probably due to the clinical severity of our patients [13,22,27-29].
This study has some limitations. First of all, it was a retrospective study. Also, because only one $\mathrm{CT}$ scan was included per patient, no CT aspect evolution analysis could be performed; however, the rapidity of evolution of symptoms might be another predictive factor of pejorative evolution. Additionally, we decided to routinely review each COVID-19 scan collectively to maximise our diagnostic value for the patients; therefore, the interobserver agreement was not studied. Finally, it is possible that some signs are not associated with pejorative evolution because of the relatively small number of deaths in our cohort. All these points would be interesting to investigate in future studies.

\section{Conclusions}

Interestingly, the presence of traction bronchiectasis and focal vascular dilatations are associated with a pejorative evolution: pulmonary fibrosis lesions could play a part in the physiopathology of COVID-19 pneumonia. High values of affected lung volume were also associated with a pejorative evolution, which concurs with the current literature on the subject.

\section{Acknowledgement}

We would like to thank our X-ray technicians for their work during this crisis.

\section{Conflict of interest}

The authors report no conflict of interest.

\section{References}

1. Zhu N, Zhang D, Wang W, et al. A novel coronavirus from patients with pneumonia in China, 2019. N Engl J Med 2020; 382: 727-733.

2. Lu H, Stratton CW, Tang Y-W. Outbreak of pneumonia of unknown etiology in Wuhan, China: the mystery and the miracle. J Med Virol 2020; 92: 401-402.

3. Xu X, Chen P, Wang J, et al. Evolution of the novel coronavirus from the ongoing Wuhan outbreak and modeling of its spike protein for risk of human transmission. Sci China Life Sci 2020; 63 : 457-460.

4. Li W, Shi Z, Yu M, et al. Bats are natural reservoirs of SARS-like coronaviruses. Science 2005; 310: 676-679.

5. COVID-19 situation update worldwide, as of 6 May 2020 [Internet]. European Centre for Disease Prevention and Control. Available from: https://www.ecdc.europa.eu/en/geographical-distribution2019-ncov-cases (Accessed: 6.05.2020).

6. Bulletin Epidémiologique Hebdomadaire - France [Internet]. Available from: www.santepubliquefrance.fr/maladies-et-traumatismes/ maladies-et-infections-respiratoires/infection-a-coronavirus/ documents/bulletin-national/covid-19-point-epidemiologiquedu-16-avril-2020 (Accesed: 20.04.2020).
7. Cheng Z, Lu Y, Cao Q, et al. Clinical features and chest CT manifestations of coronavirus disease 2019 (COVID-19) in a single-center study in Shanghai, China. AJR Am J Roentgenol 2020; 215: 121-126.

8. Zhang J, Dong X, Cao Y, et al. Clinical characteristics of 140 patients infected with SARS-CoV-2 in Wuhan, China. Allergy 2020; 75: 1730-1741.

9. He JL, Luo L, Luo ZD, et al. Diagnostic performance between CT and initial real-time RT-PCR for clinically suspected 2019 coronavirus disease (COVID-19) patients outside Wuhan, China. Respir Med 2020; 168: 105980.

10. Ai T, Yang Z, Hou H, et al. Correlation of chest CT and RT-PCR testing in coronavirus disease 2019 (COVID-19) in China: a report of 1014 cases. Radiology 2020; 296: 200642.

11. Xiong Y, Sun D, Liu Y, et al. Clinical and high-resolution CT features of the COVID-19 infection: comparison of the Initial and follow-up changes. Invest Radiol 2020; 55: 332-339.

12. Zhou Z, Guo D, Li C, et al. Coronavirus disease 2019: initial chest CT findings. Eur Radiol 2020; 30.

13. Zhou S, Wang Y, Zhu T, et al. CT features of coronavirus disease 2019 (COVID-19) pneumonia in 62 patients in Wuhan, China. AJR Am J Roentgenol 2020; 214: 1287-1294. 
14. Yuan M, Yin W, Tao Z, et al. Association of radiologic findings with mortality of patients infected with 2019 novel coronavirus in Wuhan, China. PLoS One 2020; 15: e0230548.

15. Zhao W, Zhong Z, Xie X, et al. Relation between chest CT findings and clinical conditions of coronavirus disease (COVID-19) pneumonia: a multicenter study. AJR Am J Roentgenol 2020; 214: 1072-1077.

16. Yang R, Li X, Liu H, et al. Chest CT severity score: an imaging tool for assessing severe COVID-19. Radiol Cardiothorac Imaging 2020; 2: e200047.

17. Wu Z, McGoogan JM. Characteristics of and important lessons from the coronavirus disease 2019 (COVID-19) outbreak in China: summary of a report of 72314 cases from the chinese center for disease control and prevention. JAMA 2020; 323: 1239-1242.

18. Clinical management of severe acute respiratory infection when COVID-19 is suspected [Internet]. Available from: https://www.who. int/publications-detail/clinical-management-of-severe-acute-respiratory-infection-when-novel-coronavirus-(ncov)-infection-is-suspected (Accessed: 3.05.2020).

19. Hansell DM, Bankier AA, MacMahon H, et al. Fleischner Society: glossary of terms for thoracic imaging. Radiology 2008; 246: 697-722.

20. Compte-rendu TDM THORACIQUE IV [Internet]. SFR e-Bulletin. 2020. Available from: https://ebulletin.radiologie.fr/actualit\%C3\%A9scovid-19/compte-rendu-tdm-thoracique-iv (Accessed: 7.07.2020).

21. Zare Mehrjardi M, Kahkouee S, Pourabdollah M. Radio-pathological correlation of organizing pneumonia (OP): a pictorial review. Br J Radiol 2017; 90: 20160723.

22. Ye Z, Zhang Y, Wang Y, et al. Chest CT manifestations of new coronavirus disease 2019 (COVID-19): a pictorial review. Eur Radiol 2020; 30: 4381-4389.

23. Zhang N, Xu X, Zhou L-Y, et al. Clinical characteristics and chest CT imaging features of critically ill COVID-19 patients. Eur Radiol 2020; 30: 6151-6160.

24. Feng Y, Ling Y, Bai T, et al. COVID-19 with different severities: a multicenter study of clinical features. Am J Respir Crit Care Med 2020; 201: 1380-1388.

25. Copin M-C, Parmentier E, Duburcq T, et al. Time to consider histologic pattern of lung injury to treat critically ill patients with COVID-19 infection. Intensive Care Med 2020; 46: 1124-1126.

26. Wong KT, Antonio GE, Hui DSC, et al. Thin-section CT of severe acute respiratory syndrome: evaluation of 73 patients exposed to or with the disease. Radiology 2003; 228: 395-400.

27. Liu K-C, Xu P, Lv W-F, et al. CT manifestations of coronavirus disease-2019: a retrospective analysis of 73 cases by disease severity. Eur J Radiol 2020; 126: 108941.

28. Guan CS, Lv ZB, Yan S, et al. Imaging features of coronavirus disease 2019 (COVID-19): evaluation on thin-section CT. Acad Radiol 2020; 27: 609-613.

29. Li Y, Xia L. Coronavirus disease 2019 (COVID-19): role of chest CT in diagnosis and management. AJR Am J Roentgenol 2020; 214: 1280-1286. 